\title{
CYTOTOXIC T LYMPHOCYTES DIRECTED AGAINST DONOR HLA CLASS I ANTIGENS ON AIRWAY EPITHELIAL CELLS ARE PRESENT IN BRONCHOALVEOLAR LAVAGE FLUID FROM LUNG TRANSPLANT RECIPIENTS DURING ACUTE REJECTION
}

Jun Nakajima, MD

Nancy J. Poindexter, $\mathrm{PhD}^{\mathrm{b}}$

Peter B. Hillemeyer, BS ${ }^{c}$

Elbert P. Trulock, $\mathrm{MD}^{\mathrm{d}}$

Joel D. Cooper, $\mathrm{MD}^{\mathrm{c}}$

G. Alexander Patterson, $\mathrm{MD}^{\mathrm{c}}$

T. Mohanakumar, $\mathrm{PhD}^{\mathrm{b}}$

R. Sudhir Sundaresan, MD $^{c}$
Background: The lung epithelium is among the first donor tissues encountered by the lung allograft recipient's immune system. The purpose of this study was to determine whether lung epithelium was recognized by T lymphocytes that are isolated from bronchoalveolar lavage fluid of lung allograft recipients during periods of acute rejection. Methods: Lymphocytes isolated from 45 bronchoalveolar lavage samples (from 41 lung transplant recipients) served as effector cells in standard cell-mediated cytolytic assays with several cell lines as targets: BEAS-2B (an immortalized airway epithelial cell line); B-lymphoblastoid cell lines; and $\mathbf{K 5 6 2}$ (a natural killer-sensitive cell line). Cytotoxic T-lymphocyte activity of bronchoalveolar lavage lymphocytes was correlated with pathologic status. Results: During acute rejection alone (ie, without concomitant cytomegalovirus infection), mean lysis of the airway epithelial target was significantly greater, compared with during no rejection, when these targets expressed donor-specific HLA class I antigens $(P=$ .007). Lysis of donor class I-matched B-lymphoblastoid cell line targets during rejection was not significantly different from lysis during norejection periods $(P=.18)$. Mean lysis of $\mathrm{K562}$, a natural killer cell target, did not differ between acute rejection (without concomitant cytomegalovirus infection) and no rejection $(P=.30)$. During cytomegalovirus infection (without concomitant acute rejection), there was no difference in mean lysis of airway epithelial cells, B-lymphoblastoid cell lines, or K562 targets compared with during no cytomegalovirus infection, whereas during acute rejection, compared with cytomegalovirus infection without rejection, there was a significant increase in mean lysis of the airway epithelial target when it expressed donor-specific HLA antigens $(P=.01)$. Conclusions: Donor HLA class I-specific cytotoxic T-lymphocyte activity directed at airway epithelial cells was demonstrated in bronchoalveolar lavage lymphocytes from lung transplant recipients. Lysis of these targets was significantly higher during episodes of acute rejection. (J Thorac Cardiovasc Surg 1999; 117:565-71)
$\mathrm{D}$ onor-specific cytotoxic T lymphocytes (CTL) are considered to play an important role in acute allograft rejection by virtue of their direct cytotoxicity against the graft parenchymal cells. A primed lymphocyte test (PLT) response ${ }^{1,2}$ and donor-specific CTL

From the Division of Cardiothoracic Surgery, ${ }^{\mathrm{a}, \mathrm{c}}$ Department of Surgery and Pathology, ${ }^{b}$ and the Pulmonary and Critical Care Division ${ }^{\mathrm{d}}$ Department of Medicine, Washington University School of Medicine, Barnes Hospital, St Louis, Mo.

This work was supported by National Institutes of Health grant HL56643 (T.M.).

Received for publication Aug 3, 1998; revisions requested Sept 17, activity ${ }^{3,4}$ in heart-lung or double-lung transplant recipients have been previously demonstrated. In all of these studies, donor peripheral blood lymphocyte (PBL) or lymphoblastoid cells have been used as stimulator cells. Because the lung epithelium is one of the first tissues

1998; revisions received Oct 12, 1998; accepted for publication Oct 12, 1998.

Address for reprints: Sudhir Sundaresan, MD, Northwestern University Medical School, 251 East Chicago Ave, Suite 1030, Chicago, IL 60611.

Copyright @ 1999 by Mosby, Inc.

$0022-5223 / 99 \$ 8.00+0 \quad \mathbf{1 2} / \mathbf{1} / \mathbf{9 5 1 5 7}$ 
Table I. Targets used in CTL analysis

\begin{tabular}{lll}
\hline Cell line & Type & \multicolumn{1}{c}{ HLA } \\
\hline BEAS-2B & AECL & A2,28;B45,60;DR2,3 \\
VAVY & LCL* & A1,-;B8,-;DR3,- \\
EAG254 & LCL & A3,24;B7,62;DR2,4 \\
EBZ369 & LCL & A2,-;B7,44;DR4,15 \\
K562 & NK sensitive & HLA negative \\
\hline
\end{tabular}

$\overline{A E C L}, \mathrm{SV} 40-$ transfected airway epithelial cell line ${ }^{10} ; L C L$, lymphoblastoid cell lines; $N K$, natural killer.

"Epstein Barr-transformed B-lymphoblastoid cell line.

encountered by the recipient's immune system, we hypothesized that these cells should be the target of recipient cells in in vitro assays. The purpose of this study was to demonstrate the presence of donor major histocompatibility complex (MHC) directed CTL in bronchoalveolar lavage (BAL) fluid lymphocyte populations of lung transplant recipients, to determine whether these cells were reactive with airway epithelial cells expressing donor mismatched class I molecules, and to correlate this reactivity with the occurrence of acute rejection. We hypothesize that, as the recipient's lymphocytes undergo allosensitization to parenchymal cells of the donor lung, ${ }^{5,6}$ the resultant alloreactive CTL should exhibit cytotoxicity against donor MHCmatched target cell lines, specifically bronchial epithelial cells.

\section{Patients and methods}

General. This study was conducted under full institutional review board approval, and all patients signed an informed consent form acknowledging their participation before entry. Forty-five BAL fluid samples were obtained from 41 lung transplant recipients for this study. HLA phenotypes of the lung transplant recipients and their respective donors were determined with the microcytotoxicity technique ${ }^{7}$ in the HLA laboratory at Barnes-Jewish Hospital. All of the transplantations were carried out with negative cross match (in retrospect), and no attempt was made to achieve prospectively an HLA match between donors and recipients. Because the main objective of the study was to demonstrate class I-directed CTL activity of BAL lymphocytes during acute rejection, BAL samples were obtained, whenever possible, from recipients such that at least one of their sensitizing donor alloantigens was represented in the panel of targets.

Immunosuppression. The immunosuppression regimen has been described elsewhere in greater detail. ${ }^{8}$ In brief, the induction regimen consisted of cyclosporine (INN: ciclosporin), azathioprine, and antithymocyte globulin (since 1992) followed by a maintenance regimen of cyclosporine, azathioprine, and prednisone. Steroids were given in the following manner: methylprednisolone $1 \mathrm{~g}$ intravenously before reperfusion, then $0.5 \mathrm{mg} / \mathrm{kg}$ intravenously daily, and convert to oral prednisone $0.5 \mathrm{mg} / \mathrm{kg}$ daily, tapered gradually to approximately $15 \mathrm{mg}$ orally daily at 1 year.

Follow-up bronchoscopy after transplantation. Recipient follow-up included interval chest radiographs, arterial blood gases, pulmonary function testing, and fiberoptic bronchoscopy. BAL and transbronchial lung biopsy (TBLB) were performed (by fiberoptic bronchoscopy) routinely at 1, 3, 6, and 12 months after transplantation then annually thereafter. Additional BAL and TBLB were carried out when there was clinical suspicion of rejection or infection. The bronchoscopic procedure has been previously described.$^{8}$ Lavage fluid was submitted for routine clinical tests, and a small leftover aliquot was provided for this study.

Pathologic diagnosis. TBLB tissue was fixed in formaldehyde solution and stained with hematoxylin and eosin to allow histologic analysis.

Diagnosis of acute rejection. Pathologic findings from TBLB were used to confirm the presence and grade of acute rejection. In brief, the grade of rejection was histologically classified according to the working formulation for the standardization of terminology in heart and lung allograft rejection set forth by the Lung Rejection Study Group ${ }^{9}$ and graded from $\mathrm{A} 0$ to $\mathrm{A} 4$.

Diagnosis of cytomegalovirus infection. Cytomegalovirus pneumonitis was identified histologically by characteristic intracellular inclusions on the hematoxylin and eosin stain or by a positive immunoperoxidase stain.

\section{Cell-mediated lymphocytolysis assay}

BAL lymphocyte preparation. Lymphocytes were isolated from BAL fluid and used as effector cells in standard cellmediated lymphocytolysis (CML) assays. BAL fluid was filtered through 4 layers of surgical gauze and centrifuged at $300 \mathrm{~g}$ for 12 minutes. The sediments were washed twice in phosphate-buffered saline solution and resuspended in $5 \mathrm{~mL}$ of culture medium consisting of RPMI-1640 (Gibco BRL, Gaithersburg, Md), $15 \%$ heat-inactivated human serum (MLC Normal Serum Male Non AB plasma; RJO Biologicals Inc, Kansas City, Mo), 5 mmol/L L-glutamine, 25 $\mathrm{mmol} / \mathrm{L}$ HEPES buffer, and $100 \mu \mathrm{g} / \mathrm{mL}$ streptomycin and $100 \mathrm{U} / \mathrm{mL}$ penicillin. Macrophages were removed by magnet sedimentation after a brief incubation with carbonyl iron. The remaining lymphocytes were purified by FicollHypaque density gradient sedimentation. In general, the volume of BAL obtained from the patient was about $100 \mathrm{~mL}$ (range, $60-110 \mathrm{~mL}$ ). The lymphocyte yield was typically about 1.0 to $2.0 \times 10^{6}$ cells per $100 \mathrm{~mL}$ of BAL (range, 0.2 $7.1 \times 10^{6}$ cells).

Target cell lines. The cell lines used included BEAS-2B (an immortalized airway epithelial cell line, which was provided by Dr Curtis Harris ${ }^{10}$; VAVY, EAG254, and EBZ369 (Epstein Barr virus-transformed B-lymphoblastoid cell lines); and the natural killer-sensitive cell line, K562. ${ }^{11}$ The HLA phenotypes of BEAS-2B and the lymphoblastoid cell lines were determined by the HLA laboratory at Barnes-Jewish Hospital (Table I). For this study, HLA-A and B were considered representative of class I, and HLA-DR was considered representative of class II. These cell lines were selected on the basis 
Table II. Pathologic status of patients who have received lung transplants

\begin{tabular}{lcc}
\hline & & $\begin{array}{c}\text { No. of assays with } \\
M H C^{+} \text {(relevant) } \\
\text { or MHC (irrelevant) } \\
\text { targets (\%) }\end{array}$ \\
\hline Pathologic diagnosis & $(n)$ & $15(83)$ \\
Quiescent $\left(\mathrm{REJ}^{-}\right)$ & 18 & $17(85)$ \\
Rejection $\left(\mathrm{REJ}^{+}\right)$ & 20 & $7(100)$ \\
Cytomegalovirus infection & 7 & \\
\hline cytomegalovirus
\end{tabular}

of their HLA phenotypes, specifically because they expressed common HLA class I antigens. For example, considering only the HLA-A locus, our 3 lymphoblastoid cell lines expressed HLA-A1, A2, A3, and A24 (Table I). By including the BEAS-2B target, HLA-A28 was also expressed. The predicted frequencies of these 5 alleles in the white population are $28.6 \%, 45.8 \%, 20.6 \%, 16.8 \%$, and $8.8 \%$, respectively. ${ }^{12}$ We therefore thought it likely that these targets would share some mismatched (or "sensitizing") donor HLA class I antigens in a substantial number of the experiments.

In summary, in each CML assay, there were 5 targets: 4 targets with MHC expression (3 lymphoblastoid cell lines and BEAS-2B) and K562, which lacks MHC expression.

Determination of target lysis. CTL analysis was performed with a standard chromium-51 release assay, as previously described. ${ }^{13}$ In brief, $0.5 \times 10^{6}$ cells serving as targets were labeled with $125 \mu \mathrm{Ci}$ of ${ }^{51} \mathrm{Cr}$ (ICN, Costa Mesa, Calif) for 60 minutes at $37^{\circ} \mathrm{C}$. After extensive washing, 2500 targets in 0.1 $\mathrm{mL}$ culture medium were transferred to wells of 96-well Ubottomed plates. BAL lymphocytes in $0.1 \mathrm{~mL}$ culture medium were co-cultured with ${ }^{51} \mathrm{Cr}$-labeled target cells for 4 hours at $37^{\circ} \mathrm{C}$. The yield of lymphocytes from BAL fluid usually gave an effector-to-target ratio between 10:1 and 20:1 (range, 6:1-40:1). After a 4-hour incubation, culture supernatants were harvested and counted with a gamma counter (Pharmacia LKB Biotechnology, Uppsala, Sweden).

\section{Data analysis}

Pathologic diagnosis. In each CML assay, the pathologic diagnosis was determined from the TBLB obtained simultaneously. We sought the following histologic states, as they defined the 3 study groups: quiescent (REJ'; ie, implying no rejection or cytomegalovirus infection); acute rejection alone (REJ'; ie, without concomitant cytomegalovirus infection); and cytomegalovirus infection alone (cytomegalovirus ${ }^{+}$; ie, without concomitant acute rejection).

Mean lysis values and statistical comparisons. Mean $( \pm$ SEM) values of target lysis were compared for each target (BEAS-2B, lymphoblastoid cell lines, K562) between the following groups: quiescent versus acute rejection; quiescent versus cytomegalovirus infection; and acute rejection versus cytomegalovirus infection. T test with Dunn-Sidak adjusted probabilities and analysis of variance with Tukey's multiple comparisons test were used to determine whether there were significant differences between mean values.

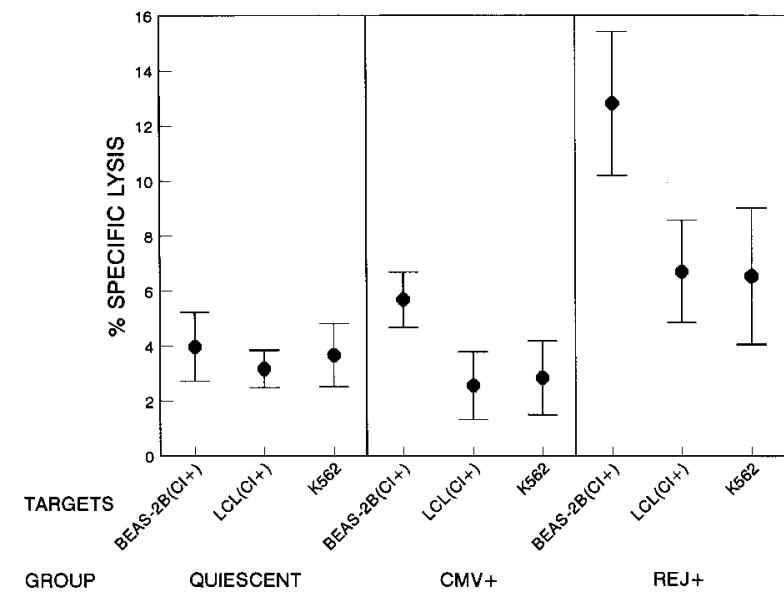

Fig 1. CTL activity of BAL lymphocytes from patients with cytomegalovirus infection and no rejection (cytomegalovirus $^{+} ; \mathrm{n}=7$ patients) are compared with quiescent patients (no rejection or cytomegalovirus infection; $\mathrm{n}=18$ patients) and patients with acute rejection and no cytomegalovirus infection $\left(\mathrm{REJ}^{+} ; \mathrm{n}=20\right.$ patients). All values represent mean \pm standard error of the mean.

Subgrouping of lysis data for the comparison of quiescent versus acute rejection. For comparison between quiescent versus acute rejection, the mean values for target lysis were subgrouped as $\mathrm{Cl} \mathrm{I}^{+}$or $\mathrm{Cl} \mathrm{II}^{+}$and $\mathrm{Cl}^{-}$, respectively, to determine the effect of MHC restriction on the lysis. This was accomplished as follows: each CML assay yielded a lysis value for the 5 targets. For those targets with MHC expression (BEAS-2B and the 3 lymphoblastoid cell lines), the target could be categorized as $\mathrm{Cl} \mathrm{I}^{+}$or $\mathrm{Cl} \mathrm{II}^{+}$, if it shared a mismatched class I or II antigen with that recipient's donor, or as $\mathrm{Cl}^{-}$, if it lacked entirely any mismatched donor class I or II antigens.

Mean values for K562 lysis cannot be subgrouped this way because this cell line lacks MHC expression. For the comparison between the cytomegalovirus ${ }^{+}$versus quiescent and cytomegalovirus ${ }^{+}$versus $\mathrm{REJ}^{+}$groups, the mean values for target lysis were subgrouped as $\mathrm{Cl}^{+}$, if they shared a mismatched class I or II antigen with that recipient's donor. Class I and II were not separated because of the small numbers of CML assays conducted on cytomegalovirus ${ }^{+}$recipients (Fig 1).

\section{Results}

General. The bronchoscopies providing samples for this study were conducted at a mean interval of 10.5 months after transplantation (range, 1-36 months).

Pathologic status and distribution of $\mathrm{Cl} \mathrm{I}^{+}, \mathrm{Cl} \mathrm{II}^{+}$, and $\mathrm{Cl}^{-}$targets. Pathologic status of the recipients is summarized in Table II. There were 18 patients in the quiescent group (no rejection or cytomegalovirus infection); 20 patients in the $\mathrm{REJ}^{+}$group (acute rejection 


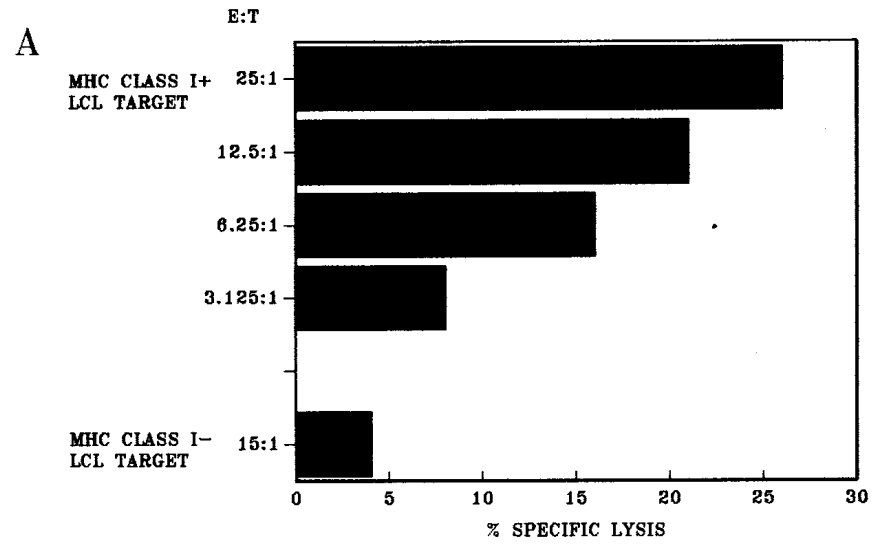

B

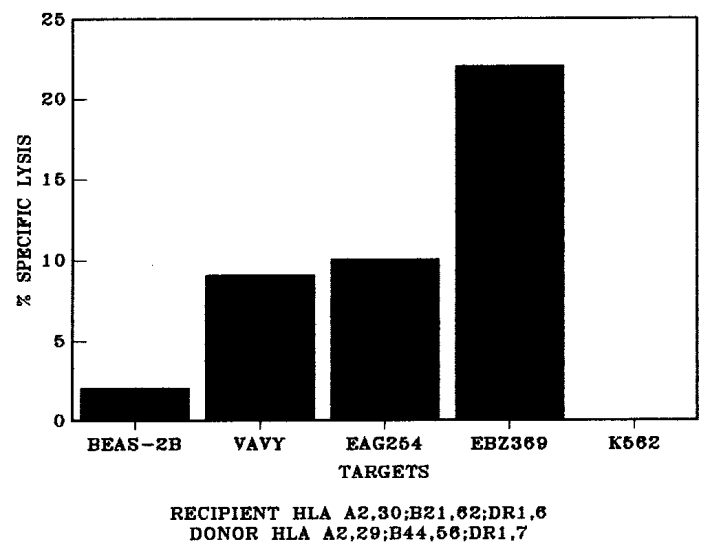

$\mathrm{C}$

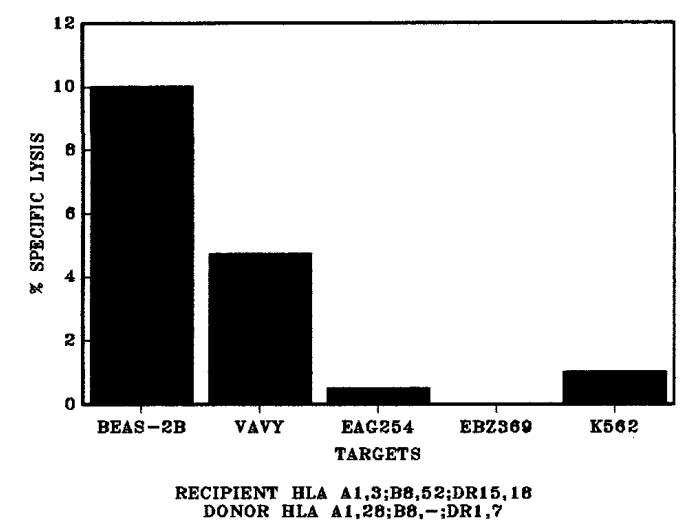

Fig 2. Examples of lysis data from CML assays. BAL lymphocytes (A) were used at several effector-to-target

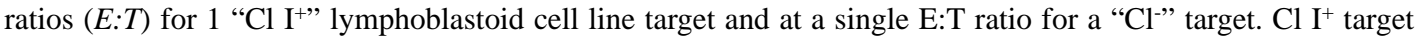
has at least 1 mismatched donor class I antigen; $\mathrm{Cl}^{-}$target lacks any mismatched donor class I or II antigens. B and $\mathbf{C}$, HLA class I $(H L A-A, B)$ and class II $(H L A-D R)$ phenotypes of recipient and donor that pertain to two assays from the acute rejection group are shown. The recipient (B) was exposed to alloantigens HLA-A29 and B44, or HLA-A28 in C. Phenotypes of the targets used are shown in Table I.

without cytomegalovirus infection); and 6 patients in the cytomegalovirus ${ }^{+}$group (cytomegalovirus infection without rejection). In 15 of the 18 assays (83\%) in the quiescent group at least 1 target of those tested shared mismatched donor class I or II antigens. In 17 of the 20 assays (85\%) in the acute rejection group, at least 1 target shared mismatched donor class I or II antigens. Finally, in 7 of the 7 assays $(100 \%)$ in the cyto- 
megalovirus $^{+}$group, at least 1 target shared mismatched donor class I or II antigens.

Allospecific CTL activity. Before embarking on this study, several preliminary CML assays were performed to evaluate its feasibility. In these assays, BAL lymphocytes were obtained from recipients proved to be undergoing acute rejection, and only 2 targets were used in the panel: $1 \mathrm{Cl} \mathrm{I}^{+}$lymphoblastoid cell lines target (ie, lymphoblastoid cell lines containing at least 1 mismatched donor class I antigen) and $1 \mathrm{Cl}^{-}$lymphoblastoid cell lines target (ie, no mismatched donor class I or II antigen shared by the lymphoblastoid cell lines). Data from 1 such assay (Fig 2, A) shows specific lysis of the $\mathrm{Cl} \mathrm{I}^{+}$target; the $\mathrm{Cl}^{-}$target was not lysed by the BAL T cells. This effect was titratable (ie, dependent on the effector to target ratio). These results indicate that the lysis represents allospecific CTL activity.

Because preliminary experiments demonstrated the feasibility of the assays, the study was undertaken with the full panel of 5 targets in each case. Illustrative CML data from 2 recipients with acute rejection but no cytomegalovirus infection are shown in Fig 2, $B$ and $C$, respectively. In each case, BAL lymphocytes lysed the $\mathrm{Cl} \mathrm{I}^{+}$target at least twice as much as any of the $\mathrm{Cl}^{-}$targets. In 1 of the 2 patients (Fig 2,C), the airway epithelial cell BEAS-2B was lysed. This again demonstrates CTL activity directed at targets bearing donor MHC class I alloantigens.

Relationship between CTL activity, acute rejection, and MHC class I and II antigens. Mean percent lysis ( \pm SEM) of BEAS-2B, lymphoblastoid cell lines, and K562 targets was compared between the acute rejection $\left(\mathrm{REJ}^{+}\right)$versus quiescent (no rejection $\left[\mathrm{REJ}^{-}\right]$) groups (Fig 3). For the BEAS-2B target, mean lysis was significantly greater during rejection compared with no rejection when the target expressed donor class I alloantigens $(11.1 \% \pm 1.9 \%[\mathrm{n}=9]$ vs $2.1 \% \pm 1.2 \%$ [n $=4] ; P=.007)$. A difference was noted between rejection and no-rejection groups when the BEAS-2B target expressed class II donor alloantigens $(15.3 \% \pm$ $3.9 \%[\mathrm{n}=7]$ vs $5.0 \% \pm 1.8 \%[\mathrm{n}=7] ; P=.09)$ and even when BEAS-2B did not share any donor class I or II allo-antigens $(13.6 \% \pm 4.5 \%[\mathrm{n}=8]$ vs $2.3 \% \pm 0.7 \%[\mathrm{n}$ $=7] ; P=.09$ ). When $\mathrm{Cl} \mathrm{I}^{+}$lymphoblastoid cell lines targets were tested even when they expressed donor alloantigens the lysis during rejection was not significantly greater than the no-rejection group $(8.2 \% \pm$ $2.5 \%[\mathrm{n}=24]$ vs $3.0 \% \pm 0.9 \%[\mathrm{n}=21] ; P=.18)$. Mean lysis did not differ between rejection and no-rejection groups for any of the other targets including $\mathrm{Cl} \mathrm{II}^{+}$ lymphoblastoid cell lines $(P=.30), \mathrm{Cl}^{-}$lymphoblastoid cell lines targets $(P=.58)$, or K562 targets $(P=.30)$.

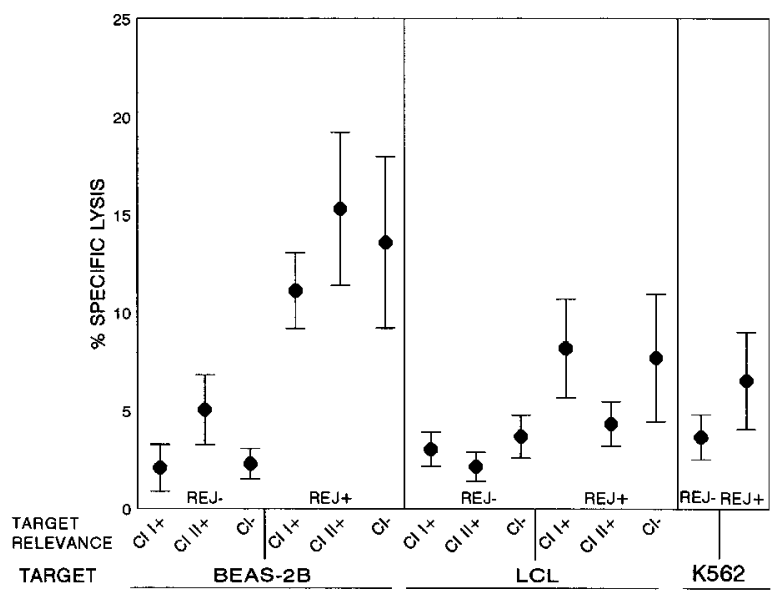

Fig 3. CTL activity of BAL lymphocytes from patients who underwent acute rejection alone $\left(\mathrm{REJ}^{+}\right)$compared with the quiescent patients $\left(\mathrm{REJ}^{-}\right) . \mathrm{Cl} \mathrm{I}^{+}$and $\mathrm{Cl} \mathrm{II}^{+}$targets express at least 1 mismatched donor antigen. $\mathrm{Cl}^{-}$targets lack any donor mismatched antigens. The number of patients in each target group range from 3 to 23, depending on the number of donormatched antigens on each of the 4 targets used in each assay. All values represent mean \pm standard error of the mean.

In summary, there was greater lysis of the airway epithelial target during acute rejection when this target shared 1 or more donor class I (sensitizing) alloantigens. However, there was also greater lysis of the airway epithelial targets, although not statistically greater, during acute rejection even when these did not share any sensitizing donor class I alloantigens.

Relationship between cytotoxicity and cytomegalovirus infection. To determine whether cytomegalovirus infection had any effect on donor-specific responses, we looked at target lysis in patient samples identified as cytomegalovirus positive. For this analysis we compared the lysis of targets that expressed donor mismatched MHC class I and II antigens together $\left(\mathrm{Cl}^{+}\right)$. When the mean percent lysis of the BEAS-2B, lymphoblastoid cell lines, and K562 targets were compared with the use of a 3-way analysis of variance between the quiescent versus cytomegalovirus ${ }^{+}$versus acute rejection, a significant difference in mean lysis was noted only between the acute rejection group and the cytomegalovirus ${ }^{+}$group when the airway epithelial cell expressing donor mismatched MHC class I or II antigens was used as a target $(12.8 \% \pm 2.6 \%[\mathrm{n}=12]$ vs $5.68 \% \pm 1.0 \%[\mathrm{n}=5] ; P=.012)$.

\section{Discussion}

Our data demonstrate the presence of donor $\mathrm{MHC}$ class I-directed airway epithelial cell CTL activity in 
the BAL lymphocyte population from patients with lung transplants during acute rejection. A number of previous studies have reported on the behavior of BAL lymphocytes isolated from pulmonary allografts, both experimentally ${ }^{4}$ and clinically. ${ }^{1-3}$ BAL, being a relatively noninvasive means of sampling the cellular and soluble components of the airways, logically has been used to study the cellular immune processes related to pulmonary allograft rejection. Donor antigen-specific PLT alloreactivity of BAL lymphocytes has been shown to correlate with both acute rejection ${ }^{1}$ and posttransplantation obliterative bronchiolitis. ${ }^{2}$ Rabinowich and coworkers ${ }^{3}$ isolated lymphocytes from TBLB tissue fragments, cultured them in media containing interleukin-2, and showed that these tissue-infiltrating lymphocytes and BAL lymphocytes demonstrated allospecific cytotoxicity in acute rejection and obliterative bronchiolitis. These findings suggested that the behavior of BAL lymphocytes correlate well with the in situ immune status of the allograft. ${ }^{3}$ Reinsmoen and colleagues ${ }^{14}$ used a panel of homozygous typing cells of known HLA antigens to further characterize the class I or II restriction of the PLT response and reported a predominantly class II-directed PLT response in acute rejection and a predominantly class I-directed pattern of PLT response in obliterative bronchiolitis. ${ }^{14}$ Thus there is strong evidence that a PLT alloproliferative response, analogous to a secondary mixed leukocyte reaction, is demonstrable in acutely rejecting lung allografts, implying the presence of activated $\mathrm{T}$ cells in the graft.

Unlike the previously mentioned studies that have focused on the proliferative response of graft-derived lymphocytes to donor antigens, our study attempted to evaluate the immune status of the graft through demonstration of donor antigen-specific cytotoxicity with CML assays with BAL lymphocytes, specifically examining the activity against an airway epithelial cell. Our demonstration of increased lysis of donor class I-relevant targets during rejection strongly suggests the presence of allospecific CTL in lung allografts during rejection. Recognizing the importance of the role of CTLs in acute cellular allograft rejection, our study would appear to most directly demonstrate ongoing rejection in vivo that is targeted to the epithelium.

A unique feature of our study was that we used an airway epithelial cell line, BEAS-2B, as a target in the panel. There was significantly greater lysis of BEAS2B during acute rejection compared with the "quiescent" state. However, unlike the lymphoblastoid cell line targets, against which the slight increase in cytotoxicity during rejection was confined to donor class
I-relevant targets, an increased lysis of BEAS-2B occurred regardless of whether or not BEAS-2B shared any class I or II antigens with the donor. But, the increased lysis reached significant levels $(P=.007)$ only when the BEAS-2B target expressed the donor class I alloantigen. Hadley and colleagues ${ }^{15}$ previously reported on clones of human CTL that exhibit class I allospecificity in a kidney tissue-restricted manner, with an in vitro mixed leukocyte-kidney culture system. The basis for the tissue specificity displayed by those clones was shown to be tissue-specific peptides presented in the context of allo-class I. ${ }^{16}$ Our finding of elevated CTL activity against BEAS-2B during rejection raises the possibility that the CTL recognizes a unique bronchial epithelial antigen presented in the context of MHC class I. This hypothesis can be tested only when cloned $\mathrm{T}$ cells are available. We are currently conducting further investigation to answer this question.

Cytomegalovirus infection and acute rejection can share similar clinical presentations in patients with lung transplants. Previous investigators have used proliferative assays to identify cytomegalovirus infection in lung allografts. These investigators have made correlations between cytomegalovirus infection and BAL lymphocyte proliferation in response to cytomegalovirus antigens, ${ }^{17}$ donor-specific MHC antigens, ${ }^{2}$ and even autologous (recipient) MHC antigens. ${ }^{14}$ Our study showed no difference in target lysis between the cytomegalovirus $^{+}$and histologically normal groups whereas there was a significant difference between target lysis, BEAS-2B sharing donor-mismatched class I or II antigens, in the cytomegalovirus ${ }^{+}$and acute rejection group. Notwithstanding the proliferative responses of cytomegalovirus-primed BAL lymphocytes described by other investigators, ${ }^{2,5,17}$ the CML assay system we describe appears to distinguish reliably between acute rejection and cytomegalovirus infection only when airway epithelial cell was used as a target.

We included K562 as a target in the panel to evaluate the potential contribution of natural killer cells to lung allograft rejection in vivo. Our data show that although natural killer cell activity was evident in the BAL cell population, it is unlikely that it plays an important role in pulmonary allograft rejection, because there was no significant difference in natural killer activity between acute rejection, cytomegalovirus infection, and normal activity. It is possible that the use of cyclosporine masked the importance of these natural killer cells by virtue of its potent inhibitory effects on interleukin-2 release. Further work would be necessary to clarify this observation.

In summary, donor class I-directed CTL activity is 
present in BAL lymphocytes from patients with lung transplants who are undergoing acute rejection. The activity is most apparent when the airway epithelial cell is used as a target. These results suggest that a major component of the immune response to lung allograft is directed against the lung epithelium. These results may have direct implications on the possible development of chronic responses in these patients. Destruction of the epithelium is a major pathologic feature in patients with bronchiolitis obliterans syndrome. Our results suggest that these changes may be immune mediated.

We thank Karen McBride and David Misselhorn, whose consistent and conscientious help allowed the clinical samples for this study to be obtained; Richard B. Schuessler, $\mathrm{PhD}$, for his help with statistical analysis of data; and Billie Glasscock for assistance in preparing this manuscript.

\section{REFERENCES}

1. Zeevi A, Fung JJ, Paradis IL, et al. Lymphocytes of bronchoalveolar lavage from heart-lung transplant recipients. J Heart Transplant 1985;4:417-21.

2. Rabinowich H, Zeevi A, Paradis I. Proliferative responses of lavage lymphocytes from heart-lung patients. Transplantation 1990;49:115-21.

3. Rabinowich H, Zeevi A, Yousem SA, et al. Alloreactivity of lung biopsies and bronchoalveolar lavage-derived lymphocytes from pulmonary transplant patients: correlation with acute rejection and bronchiolitis obliterans. Clin Transplant 1990;4:376-84.

4. Kirby JA, Parfett GJ, Reader JA, Pepper JR. Lung transplantation in the rat: a model for study of the cellular mechanisms of allograft rejection. Immunology 1988;63:369-72.

5. Snell G. The homograft reaction. Ann Rev Microbiol 1957; 11:439-63.

6. Steinmuller D. Immunization with skin allografts taken from tolerant mice. Science 1967;158:127-9.

7. NIH Transplantation Immunology Branch Staff. Lymphocyte cytotoxicity. In: NIAID Manual of Tissue Typing Techniques, 1976-1977. Rockville [MD]: NIH; 1978. p. 22.

8. Trulock EP, Ettinger NA, Brunt EA, Pasque MK, Kaiser LR, Cooper JD. The role of transbronchial lung biopsy in the management of lung transplant recipients: an analysis of 200 consecutive procedures. Chest 1992;102:1049-54.

9. Berry GJ, Brunt EM, Chamberlain D, et al. A working formulation for the standardization of nomenclature in the diagnosis of heart and lung rejection: lung rejection study group: the International Society for Heart Transplantation. J Heart Transplant 1990;9:593-601.

10. Reddel RR, Ke Y, Gerwin BI, et al. Transformation of human bronchial epithelial cells by infection with SV40 or adenovirus12 SV40 hybrid virus, or transfection via strontium phosphate coprecipitation with a plasmid containing SV40 early region genes. Cancer Res 1988;48:1904-9.

11. Fitzgerald PA, Evans R, Kirkpatrick D, Lopez C. Heterogeneity of human NK cells: comparison of effectors that lyse HSV-1 infected fibroblasts and K562 erythroleukemia targets. J Immunol 1983;130:1663-7.

12. The Central Data Analysis Committee. Allele frequencies, section 6.3 splits combines (five loci). In: The data book of the 11th International Histocompatibility Workshop. Yokohama, Japan: 1991. p. 807.

13. Hadley GA, Linders B, Mohanakumar T. Immunogenicity of MHC class I alloantigens expressed on parenchymal cells in the human kidney. Transplantation 1992;54:537-42.

14. Reinsmoen NL, Bolman RM, Savik K, Butters K, Hertz M. Differentiation of class I- and class II-directed donor-specific alloreactivity in bronchoalveolar lavage lymphocytes from lung transplant recipients. Transplantation 1992;53:181-9.

15. Hadley GA, Linders B, Mohanakumar T. Kidney cell-restricted recognition of MHC class I alloantigens by human cytolytic T cell clones. Transplantation 1993;55:400-4.

16. Poindexter NJ, Naziruddin B, McCourt DW, Mohanakumar T. Isolation of a kidney-specific peptide recognized by alloreactive HLA-A3-restricted human CTL. J Immunol 1995;154:3880-7.

17. Zeevi A, Ukins ME, Spichty KJ, et al. Proliferation of cytomegalovirus-produced lymphocytes in bronchoalveolar lavages from lung transplant patients. Transplantation 1992;54:635-9. 